\title{
A UNIQUENESS THEOREM FOR THE MINIMAL SURFACE EQUATION ON AN UNBOUNDED DOMAIN IN $\mathbb{R}^{2}$
}

\author{
Chin-Chuan Lee
}

In this paper, we give a variation on Nitsche's result on solutions to the minimal surface equation in sectors.

In 1965, Nitsche [1] announced the following result:

Theorem 1. Let $\Omega$ be a sector domain of opening angle smaller than $\pi$. If $u=u(x, y) \in C^{2}(\Omega) \cap C^{0}(\bar{\Omega})$ is a solution of

$$
\begin{cases}\operatorname{div} \mathbf{T} u=0 & \text { in } \Omega \\ u=0 & \text { on } \partial \Omega\end{cases}
$$

then $u \equiv 0$ in $\Omega$. Here $\mathbf{T} u=\frac{\nabla u}{\sqrt{1+|\nabla u|^{2}}}$.

In this paper, we give a variation of Theorem 1 as follows:

Theorem 2. Let $\Omega$ be a sector domain of opening angle smaller than $\pi$. If $u=u(x, y) \in C^{2}(\Omega) \cap C^{1}(\bar{\Omega} \backslash\{$ vertex $\})$ is a solution of

$$
\begin{cases}\operatorname{div} \mathbf{T} u=0 & \text { in } \Omega \\ \mathbf{T} u \cdot \nu=0 & \text { on } \partial \Omega \backslash\{\text { vertex }\}\end{cases}
$$

where $\nu$ is the exterior unit normal on $\partial \Omega \backslash\{$ vertex $\}$ then $u \equiv$ constant in $\Omega$.

Proof. First, we notice that, although we make no assumptions at the vertex, in fact, we can show that $u \in C^{2}(\Omega) \cap C^{1}(\bar{\Omega})$ as follows: Denote by $\mathrm{m}$ and $\mathrm{M}$ the lower and upper bounds for $\mathrm{u}$ on a fixed arc separating the vertex from infinity. Then, by Theorem 5.1 of [2] the two planes $v=m$ and $v=M$ lie respectively below and above $u$ in the entire domain between the vertex and the arc, and thus $\mathrm{u}$ is bounded above and below at the vertex. It then follows directly from Simon's theorem [5], that $\mathrm{u}$ is $C^{1}$ to the vertex. Thus 
$u \in C^{2}(\Omega) \cap C^{1}(\bar{\Omega})$. Since every solution $u \in C^{2}(\Omega) \cap C^{1}(\bar{\Omega})$ of $\operatorname{div} \mathbf{T} u=0$ satisfies

$$
\left\{\begin{array}{l}
\frac{\partial}{\partial x}\left(\frac{1+q^{2}}{w}\right)=\frac{\partial}{\partial y}\left(\frac{p q}{w}\right) \\
\frac{\partial}{\partial x}\left(\frac{p q}{w}\right)=\frac{\partial}{\partial y}\left(\frac{1+p^{2}}{w}\right)
\end{array}\right.
$$

where $p=\frac{\partial u}{\partial x}, q=\frac{\partial u}{\partial y}, w=\sqrt{1+p^{2}+q^{2}}$, there exist functions $F(x, y)$ and $G(x, y)$ such that

$$
\left\{\begin{array}{l}
\frac{\partial F}{\partial x}=\frac{1+p^{2}}{w}, \frac{\partial F}{\partial y}=\frac{p q}{w} \\
\frac{\partial G}{\partial x}=\frac{p q}{w}, \quad \frac{\partial G}{\partial y}=\frac{1+q^{2}}{w}
\end{array}\right.
$$

If we set

$$
\left\{\begin{array}{l}
\xi=x+F(x, y) \\
\eta=y+G(x, y)
\end{array}\right.
$$

then $\xi, \eta$ are isothermal coordinates. (c.f. $[\mathbf{3}$, p. 31])

We will show that the image of $\bar{\Omega}$ under the transformations $\xi, \eta$ is again a sector domain of opening angle smaller than $\pi$. ( $\bar{\Omega}$ is the closure of $\Omega$ ). Define a mapping $\psi: \bar{\Omega} \longrightarrow \mathbb{R}^{2}$ by $\psi(x, y)=(\xi, \eta)$, where $\xi, \eta$ are defined by (2). Without loss of generality, we may assume $\Omega$ is symmetric with respect to the y-axis. If $\langle a, b\rangle$ is the unit tangent vector of the right side of $\partial \Omega$ (that is, $\left.a^{2}+b^{2}=1 ; a, b>0\right)$ and $s$ is the arc-length function of $\partial \Omega$, then

$$
\left\{\begin{array}{l}
\frac{\partial \xi}{d s}=\left\langle\xi_{x}, \xi_{y}\right\rangle \cdot\langle a, b\rangle=a+\frac{a+p^{2} a+p q b}{w} \\
\frac{\partial \eta}{d s}=\left\langle\eta_{x}, \eta_{y}\right\rangle \cdot\langle a, b\rangle=\frac{p q a+b+b q^{2}}{w}+b
\end{array} \text { on the right side of } \partial \Omega .\right.
$$

Since $\mathbf{T} u \cdot \nu=0$ on $\partial \Omega$, we have $\left\langle\frac{p}{w}, \frac{q}{w}\right\rangle \cdot\langle b,-a\rangle=0$ on the right side of $\partial \Omega$, which implies $p b=q a$ on the right side of $\partial \Omega$. Substituting this into $\frac{d \xi}{d s}, \frac{d \eta}{d s}$, we obtain

$$
\left\{\begin{array}{l}
\frac{d \xi}{d s}=a(1+w) \\
\frac{d \eta}{d s}=b(1+w)
\end{array} \text { on the right side of } \partial \Omega .\right.
$$

Since $1+w>1, \psi$ maps the right side of $\partial \Omega$ linearly into $\mathbb{R}^{2}$. For the left side of $\partial \Omega$, we have the similar result. Thus $\psi$ maps $\bar{\Omega}$ into a sector domain 
$\overline{\Omega^{\prime}}$ in $\mathbb{R}^{2}$ and $\psi$ is a one-one, onto map from $\partial \Omega$ to $\partial \Omega^{\prime}$. Next, we show that $\psi$ is a one-one, onto map from $\Omega$ to $\Omega^{\prime}$. Let $P_{0}\left(x_{0}, y_{0}\right), P_{1}\left(x_{1}, y_{1}\right)$ be two points in $\Omega$, with $\psi\left(x_{0}, y_{0}\right)=\left(\xi_{0}, \eta_{0}\right)$ and $\psi\left(x_{1}, y_{1}\right)=\left(\xi_{1}, \eta_{1}\right)$. Consider $P_{t}\left(x_{t}, y_{t}\right)=\left(x_{0}+t\left(x_{1}-x_{0}\right), y_{0}+t\left(y_{1}-y_{0}\right)\right), 0 \leq t \leq 1$. Since $\Omega$ is convex, $P_{t}\left(x_{t}, y_{t}\right) \in \Omega$.

By (1), there exists a function $E(x, y)$ such that

$$
\frac{\partial E}{\partial x}=F \quad \text { and } \quad \frac{\partial E}{\partial y}=G .
$$

Following the method of Lemma 5.1 in $[3]$, we define a function $h:[0,1] \longrightarrow$ $\mathbb{R}$ by

$$
h(t)=E\left(x_{t}, y_{t}\right)
$$

Then

$$
\begin{aligned}
h^{\prime}(t) & =\left(x_{1}-x_{0}\right) \bar{p}+\left(y_{1}-y_{0}\right) \bar{q} \\
h^{\prime \prime}(t) & =\left(x_{1}-x_{0}\right)^{2} \bar{r}+2\left(x_{1}-x_{0}\right)\left(y_{1}-y_{0}\right) \bar{s}+\left(y_{1}-y_{0}\right)^{2} \bar{l}
\end{aligned}
$$

where

$$
\begin{aligned}
\bar{p} & =\frac{\partial E}{\partial x}\left(x_{t}, y_{t}\right) \\
\bar{q} & =\frac{\partial E}{\partial y}\left(x_{t}, y_{t}\right) \\
\bar{r} & =\frac{\partial^{2} E}{\partial x^{2}}\left(x_{t}, y_{t}\right) \\
\bar{s} & =\frac{\partial^{2} E}{\partial x \partial y}\left(x_{t}, y_{t}\right) \\
\bar{l} & =\frac{\partial^{2} E}{\partial y^{2}}\left(x_{t}, y_{t}\right) .
\end{aligned}
$$

So, $h^{\prime \prime}(t)$ is a quadratic form in $\left(x_{1}-x_{0}\right)$ and $\left(y_{1}-y_{0}\right)$, and since the matrix $\left(\begin{array}{c}\bar{p} \bar{s} \\ \bar{s}\end{array}\right)$ is positive definite, we have $h^{\prime \prime}(t)>0$. This implies $h^{\prime}(t)$ is an increasing function, thus $h^{\prime}(0)<h^{\prime}(1)$, that is,

$$
\left(x_{1}-x_{0}\right)\left(\bar{p}_{1}-\bar{p}_{0}\right)+\left(y_{1}-y_{0}\right)\left(\bar{q}_{1}-\bar{q}_{0}\right)>0
$$

where

$$
\begin{aligned}
\bar{p}_{1}=\bar{p}\left(x_{1}, y_{1}\right) & \bar{p}_{0}=\bar{p}\left(x_{0}, y_{0}\right) \\
\bar{q}_{1}=\bar{q}\left(x_{1}, y_{1}\right) & \bar{q}_{0}=\bar{q}\left(x_{0}, y_{0}\right) .
\end{aligned}
$$


From (2), we have

$$
\left(x_{1}-x_{0}\right)\left(\xi_{1}-\xi_{0}\right)+\left(y_{1}-y_{0}\right)\left(\eta_{1}-\eta_{0}\right)>\left(x_{1}-x_{0}\right)^{2}+\left(y_{1}-y_{0}\right)^{2} .
$$

By the Cauchy-Schwarz inequality, we have

$$
\left(\xi_{1}-\xi_{0}\right)^{2}+\left(\eta_{1}-\eta_{0}\right)^{2}>\left(x_{1}-x_{0}\right)^{2}+\left(y_{1}-y_{0}\right)^{2} .
$$

Thus, if $\left(x_{0}, y_{0}\right) \neq\left(x_{1}, y_{1}\right)$, then $\left(\xi_{0}, \eta_{0}\right) \neq\left(\xi_{1}, \eta_{1}\right)$, so $\psi$ is a one-one map from $\Omega$ to $\Omega^{\prime}$.

To show $\psi$ is an onto map, let $\psi(\Omega)=K \subset \Omega^{\prime}$. Because $\frac{\partial(\xi, \eta)}{\partial(x, y)} \neq 0, \psi$ is locally one-one. Thus every neighborhood of $\left(\xi_{0}, \eta_{0}\right) \in K$ belongs to $K$, that is, $K$ is relatively open to $\Omega^{\prime}$. On the other hand, if $\left(\xi_{0}, \eta_{0}\right) \in \Omega^{\prime}$ is the limit of sequence of points $\left(\xi_{i}, \eta_{i}\right) \in K$, we will show $\left(\xi_{0}, \eta_{0}\right) \in K$. But $\xi_{i}=x_{i}+$ $F(x, y), \eta_{i}=y_{i}+G(x, y)$, and bounded (because $\left.\lim _{i \rightarrow \infty}\left(\xi_{i}, \eta_{i}\right)=\left(\xi_{0}, \eta_{0}\right)\right)$. By (3), we see that $\left(x_{i}, y_{i}\right)$ is also bounded. So, there exists a convergent subsequence $\left(x_{n_{i}}, y_{n_{i}}\right)$ of $\left(x_{i}, y_{i}\right)$ such that $\lim _{n \rightarrow \infty}\left(x_{n_{i}}, y_{n_{i}}\right)=\left(x_{0}, y_{0}\right)$. Now, since $\psi$ is continuous on $\bar{\Omega}$, we obtain

$$
\text { and } \begin{aligned}
\xi_{0}=\lim _{i \rightarrow \infty} \xi_{n_{i}} & =\lim _{i \rightarrow \infty}\left(x_{n_{i}}+F\left(x_{n_{i}}, y_{n_{i}}\right)\right) \\
& =x_{0}+F\left(x_{0}, y_{0}\right) \\
\eta_{0}=\lim _{i \rightarrow \infty} \eta_{n_{i}} & =\lim _{i \rightarrow \infty}\left(y_{n_{i}}+G\left(x_{n_{i}}, y_{n_{i}}\right)\right) \\
& =y_{0}+G\left(x_{0}, y_{0}\right) .
\end{aligned}
$$

Because $\psi$ is a one-one, onto map from $\partial \Omega$ to $\partial \Omega^{\prime}$, and $\psi$ is continuous on $\bar{\Omega}$ and $\left(\xi_{0}, \eta_{0}\right) \in \Omega^{\prime}$, it follows that $\left(x_{0}, y_{0}\right) \in \Omega$. Since $\psi(\Omega)=K$ and $\xi_{0}=x_{0}+F\left(x_{0}, y_{0}\right), \eta_{0}=y_{0}+G\left(x_{0}, y_{0}\right)$, we have $\left(\xi_{0}, \eta_{0}\right) \in K$. Thus $K$ is relatively closed to $\Omega^{\prime}$. Therefore, $\psi(\Omega)=K \neq \phi$ is relatively open and closed to $\Omega^{\prime}$. On the other hand, $\psi(\Omega)=K$ is connected in $\Omega^{\prime}$, which implies $\psi(\Omega)=K=\Omega^{\prime}$, so $\psi$ is an onto map from $\Omega$ to $\Omega^{\prime}$.

We have shown that the image of $\Omega$ under the transformations $\xi, \eta$ is again a sector domain $\Omega^{\prime}$ of opening angle smaller than $\pi$.

Because $\operatorname{div} \mathbf{T} u=0$, there exists a function $v(x, y)$ such that

$$
\frac{\partial v}{\partial x}=\frac{q}{w} \quad \text { and } \quad \frac{\partial v}{\partial y}=\frac{-p}{w} .
$$

Therefore, $|\nabla v|<1$, which implies $v(x, y)<\sqrt{x^{2}+y^{2}}$. Now, by (3), we have $v(\xi, \eta)<\sqrt{\xi^{2}+\eta^{2}}$, that is, $v=O(r)$, where $r=\sqrt{\xi^{2}+\eta^{2}}$.

Also

$$
\begin{aligned}
\Delta_{(\xi, \eta)} v & =0 \quad \text { in } \Omega^{\prime} \\
\left.v\right|_{\partial \Omega^{\prime}} & =\text { constant }
\end{aligned}
$$


where $\Delta_{(\xi, \eta)}$ is the Laplacian with respect to $(\xi, \eta)$.

Thus, using the Phragmén-Lindelöf theorem for harmonic function on a sector domain ( c.f. $[4$, p. 94]), we have

$$
v(\xi, \eta) \equiv \text { constant } \quad \text { in } \Omega^{\prime}
$$

this implies $\tilde{u}(\xi, \eta) \equiv$ constant in $\Omega^{\prime}$, so $u(x, y) \equiv$ constant in $\Omega$.

\section{Acknowledgements.}

The author would like to thank the advisor Professor Ai-Nung Wang for his continuous encouragements and many useful suggestions. Also, the author would like to express his gratitude to Professor Jenn-Fang Hwang for his many discussions, instructions and much help. Finally, the author would like to thank the editor Professor Finn for his many instructive suggestions and continuous help.

\section{References}

[1] J.C.C. Nitsche, On New Results in the Theory of Minimal Surfaces, Bull. Amer. Math. Soc., 71 (1965), 195-270.

[2] R. Finn, Equilibrium Capillary Surfaces, Grundlehren der mathematischen Wissenschaften 284, Springer-Verlag, 1986.

[3] R. Osserman, A Survey of Minimal Surfaces, Van Nostrand - Reinhold, New York, 1969.

[4] M.H. Protter and H.F. Weinberger, Maximal Principles in Differential Equations, Englewood N. J. Cliffs, Prentice - Hall, 1967.

[5] L. Simon, Regularity of capillary surfaces over domains with corners, Pacific J. Math, 88 (1986), 363-377.

Received March 30, 1995.

National Taiwan University

TaIPEI, TaIWAN, R.O.C.

E-mail address: cclee@math.ntu.edu.tw 